\title{
Efficacy of fluralaner spot-on solution against induced infestations with Rhipicephalus sanguineus on dogs
}

\author{
Janina Taenzler ${ }^{1 *}$, Julian Liebenberg ${ }^{2}$, Machiel Mienie ${ }^{3}$, William R. Everett ${ }^{4}$, David R. Young ${ }^{5}$, Thomas S. Vihtelic ${ }^{6}$,
} Fangshi Sun ${ }^{7}$, Eva Zschiesche ${ }^{1}$, Rainer K. A. Roepke ${ }^{1}$ and Anja R. Heckeroth ${ }^{1}$

\begin{abstract}
Background: The efficacy of fluralaner spot-on solution administered once topically against induced infestations with Rhipicephalus sanguineus was evaluated in dogs over a 12-week post-treatment period.

Methods: Six negative-controlled studies were conducted, involving a total of 112 adult dogs (57 mixed breed, 47 Beagles, eight Labradors). In each study, dogs were randomized to two groups of eight to ten dogs each. On day 0 , dogs in each treated group were topically administered fluralaner spot-on solution once at a dose of $25 \mathrm{mg} / \mathrm{kg}$ body weight, while dogs in each control group were not treated. Two days before treatment, and on days 28, 56 and 84 after treatment, all dogs were infested with approximately 50 unfed, adult Rh. sanguineus ticks (sex ratio 1:1). Ticks were removed and counted on days 2, 30 (4 weeks), 58 (8 weeks), and 86 (12 weeks) after treatment to assess efficacy.
\end{abstract}

Results: Efficacy against ticks 2 days after treatment was $91.1 \%$ (study 1), $98.4 \%$ (study 2), $100 \%$ (study 3), $97.6 \%$ (study 4), $99.6 \%$ (study 5), and $99.8 \%$ (study 6). At all other assessment time points, tick efficacy was $95.4-100 \%$. Tick reduction in all treatment groups was significant at all assessment time points $(P<0.0001)$.

Conclusions: A single topical administration of fluralaner spot-on solution provides a high level of therapeutic and persistent efficacy against $R$ h. sanguineus ticks over the subsequent 12 weeks.

Keywords: Bravecto ${ }^{\mathrm{TM}}$ Spot-on Solution, Dog, Efficacy, Fluralaner, Rhipicephalus sanguineus, Tick

\section{Background}

Rhipicephalus sanguineus, the brown dog tick, is the most widespread tick worldwide [1]. The domestic dog is the main host of this tick in both urban and rural areas $[2,3]$, but it can also occasionally infest a wide range of domestic and wild hosts, including cats, rodents, birds, and humans [4,5]. Rhipicephalus sanguineus ticks can attach everywhere on the dog, but the head (particularly the ears), interdigital spaces, back, inguinal region and axilla are among their preferred attachment sites [6]. In tropical and subtropical areas, this tick is prevalent throughout the year [6,7], whereas in temperate regions it is most active from the late spring to early autumn [7].

\footnotetext{
* Correspondence: janinataenzler@msd.de

${ }^{1}$ MSD Animal Health Innovation GmbH, Zur Propstei, 55270 Schwabenheim, Germany

Full list of author information is available at the end of the article
}

Rhipicephalus sanguineus ticks can even overwinter in the environment and infest dogs during winter in some regions of temperate climate (e.g. southeastern Oklahoma and northwestern Arkansas, United States) [6]. As known vectors of pathogen transmission as well as a cause of discomfort and blood loss for dogs, ticks and tick infestations are a serious concern for owners. Therefore, control of $R h$. sanguineus is of great medical and veterinary importance.

Over the last decade, the number of products and strategies available for tick control has increased remarkably, offering veterinarians and pet owners the option of choosing their preferred product [8]. Most of the existing commercial tick control products usually have a 4 week tick-killing and/or repellent effect via contact exposure of ticks to the treated animal [9]. An alternative of tick control in dogs is being offered by using drugs 
from the isoxazoline chemical class (i.e. fluralaner [10], afoxolaner [11] and sarolaner [12]). This alternative exerts its effect when ticks attach to the host and are exposed to the drug through feeding activity [10]. By using fluralaner spot-on solution, a second convenient route of fluralaner administration besides the oral route $\left(\right.$ Bravecto $\left.^{\mathrm{m}}{ }^{\mathrm{N}}\right)$ is being presented to veterinarians and pet owners. After topical administration, fluralaner is readily absorbed from the administration site into the skin, from where it is distributed systemically throughout the body. An uptake of the medicated tissue fluid by the Rh. sanguineus tick results in a potent tick-killing effect. Pharmacokinetic data suggest that by topical administration of fluralaner 12 weeks of efficacy against $R h$. sanguineus can be provided [13].

This paper summarizes the results of six independent studies, which evaluated the efficacy of fluralaner spot-on solution against $R h$. sanguineus ticks over a 12 -week posttreatment period.

\section{Methods}

\section{Study set-up}

Six studies (studies 1-6) were conducted in dogs using induced tick infestations with $R h$. sanguineus after single topical fluralaner (Bravecto ${ }^{\text {ma }}$ Spot-on Solution) administration. All studies were in accordance with Good Clinical Practice (VICH guideline GL9, Good Clinical Practice, EMA, 2000). Three studies were conducted in South Africa (studies 1-3) and three studies were conducted in the United States (studies 4-6). All studies were randomized and performed as either negative-controlled and partly masked (studies 1, 4-6), or open-labelled (studies 2 and 3) (Table 1). Each study was approved by the corresponding local Ethics Committee responsible for the respective CRO before study start.

In total, 112 adult dogs (57 mixed breed, 47 Beagles, eight Labradors), consisting of males and females between 0.5 and 11 years of age, and weighing between 4.3 and $35.6 \mathrm{~kg}$ before treatment, were involved in the studies. Each dog was in good health, had not been treated with any parasite control product within 2 to 3 months prior to a 7-day acclimatization period, did not harbor any ticks before treatment, and was uniquely identified by a tattoo or a microchip number.

Prior to randomization, dogs were clinically examined and weighed. The parasite susceptibility of each dog was confirmed through the infestation with approximately 50 unfed, adult $R h$. sanguineus ticks (sex ratio 1:1), followed by tick removal and count $48 \mathrm{~h}( \pm 4 \mathrm{~h})$ later.

All dogs, from every study group, harbored more than $25 \%$ of the number of originally infested ticks. Dogs were blocked by descending live tick counts and one dog in each block was randomized to two study groups (one treatment and one control group) of eight dogs (studies 2 and 3 ) or ten dogs (studies 1,4-6), using a computer generated randomization list.

All dogs were kept indoors and housed individually during the study course. They were fed a commercially obtained dry dog food at least once daily at the recommended rate; drinking water was provided ad libitum. General health observations were performed once daily throughout the study.

\section{Treatment}

On day 0 (i.e. day of treatment), dogs in the treatment group were treated with fluralaner spot-on solution (Bravecto ${ }^{\text {Tx }}$ Spot-on Solution), based on the dog's individual body weight, to achieve a dose of $25 \mathrm{mg}$ fluralaner/ $\mathrm{kg}$ body weight. Between 0.4 and $3.2 \mathrm{ml}$ of fluralaner spot-on solution/dog was administered once topically at one or more spots, depending on the administration volume, along the dog's dorsal line. During administration, the dog was standing, or lying with its back horizontally. The first spot was administered between the shoulder blades and the other spots were administered approximately $5-10 \mathrm{~cm}$ caudal of the previous spot. The hair was parted, the tip of a positive displacement pipette (studies 2 and 3) or of a disposable syringe (studies 1, 4-6)

Table 1 Study details

\begin{tabular}{|c|c|c|c|c|c|c|c|}
\hline \multirow{2}{*}{$\begin{array}{l}\text { Study set-up } \\
\text { Study number }\end{array}$} & \multirow{2}{*}{ Study site } & \multicolumn{2}{|l|}{ Animals per group } & \multirow[t]{2}{*}{ Dog breed } & \multirow[t]{2}{*}{ Weight (kg) } & \multirow[t]{2}{*}{ Age (years) } & \multirow{2}{*}{$\begin{array}{l}\text { Tick isolate } \\
\text { used }\end{array}$} \\
\hline & & Treatment group & Control group & & & & \\
\hline 1 & ClinVet (South Africa) & 10 & 10 & Mixed and Beagles & $8.0-20.2$ & $0.9-6$ & EU \\
\hline 2 & $\begin{array}{l}\text { Malelane Research Unit, } \\
\text { MSD Animal Health (South Africa) }\end{array}$ & 8 & 8 & Mongrels and Labradors & $10.7-35.6$ & $3-6$ & SA \\
\hline 3 & $\begin{array}{l}\text { Malelane Research Unit, } \\
\text { MSD Animal Health (South Africa) }\end{array}$ & 8 & 8 & Mongrels and Labradors & $11.3-29.9$ & $2-6$ & SA \\
\hline 4 & BerTek, USA & 10 & 10 & Mixed & $6.8-14.9$ & $2.4-11.3$ & USA \\
\hline 5 & $\begin{array}{l}\text { Young Veterinary Research } \\
\text { Services, USA }\end{array}$ & 10 & 10 & Beagles & $7.7-16.3$ & $5.5-6.6$ & USA \\
\hline 6 & MPI Research, USA & 10 & 10 & Beagles & $4.3-8.7$ & $\geq 0.5$ & USA \\
\hline
\end{tabular}

Abbreviations: EU Europe, SA South Africa, USA United States 
was placed vertically on the skin, and the solution was administered directly to the skin by pressing the plunger of the syringe to empty its contents. After administration, the dog was held upright for approximately $5 \mathrm{~min}$ to observe the treatment site. No run or drip-off was observed and no additional treatment solution was applied to any dog. Dogs in the control group remained untreated.

\section{Tick infestations and assessments}

Infestations using laboratory reared $R h$. sanguineus ticks were conducted on sedated dogs (study 1) or nonsedated dogs (studies 2-6) 2 days before treatment (i.e. day -2), and on days 28 (4 weeks), 56 (8 weeks), and 84 (12 weeks) after treatment. Dogs in studies 4-6 were not sedated prior to each infestation, but were placed in an infestation chamber for approximately $4 \mathrm{~h}( \pm 10 \mathrm{~min})$ to ensure that the ticks had established an infestation. After the infestation period, the dog was released back to its kennel. Dogs in studies 1-3 were infested in their kennel.

The tick isolate used in each study for each infestation was not older than 10 years and ticks were regularly added from the field (Table 1). At each infestation time point, each dog was infested with $50( \pm 4)$ viable, unfed adult ticks (sex ratio 1:1), applied directly to its fur along the dorsal rump area, which allowed the ticks to crawl into the hair coat and select an attachment site.

Tick removal and counts were performed 2 days after treatment (i.e. on day 2$)$ and then $48 \mathrm{~h}( \pm 4 \mathrm{~h})$ after each infestation (i.e. on days 2, 30, 58 and 86), and were classified as dead or alive, attached or unattached, and engorged or unengorged. In studies 1, 4, 5 and 6 personnel conducting tick classification and tick counting were masked to the treatment status of each dog.

\section{Efficacy evaluation}

The statistical analysis was performed using the software package $\mathrm{SAS}^{\oplus}$ (SAS Institute Inc., Cary, NC, USA, release 9.1.3 (study 2), 9.2 (studies 1 and 3) and 9.3 (studies 4-6). The individual dog was the experimental unit in all statistical calculations. The percentage efficacy against ticks was calculated for the treatment group at each assessment time point using geometric means with Abbott's formula:

Efficacy $(\%)=100 \times\left(\mathrm{M}_{\mathrm{C}}-\mathrm{M}_{\mathrm{T}}\right) / \mathrm{M}_{\mathrm{C}}$, where $\mathrm{M}_{\mathrm{C}}$ is the mean number of total live ticks on untreated control dogs and $\mathrm{M}_{\mathrm{T}}$ is the mean number of total live ticks on treated dogs. Log-transformed $[\ln (x+1)]$ counts of live attached ticks were used to confirm the efficacy calculation. Significant differences were assessed between the log-counts of live ticks in the treated group at each assessment time point and the log-counts of the untreated control group. Study groups were compared using a linear mixed model, which included study group as a fixed effect and block as a random effect. The two-sided level of significance was set $P \leq 0.05$.

\section{Results}

Two days after a single topical treatment with fluralaner spot-on solution, efficacy rates of $91.1 \%$ (study 1), 98.4\% (study 2), $100 \%$ (study 3), $97.6 \%$ (study 4), $99.6 \%$ (study 5), and $99.8 \%$ (study 6) were achieved against $R h$. sanguineus ticks (i.e. therapeutic efficacy). The persistent efficacy against $R h$. sanguineus ticks, at the assessment time points 30, 56 and 86 in the different studies varied between 95.4 and $100 \%$. Tick reduction in all treatment groups was significant at all assessment time points $(P<0.001)$ (Table 2$)$.

Adequate tick infestations (at least six animals with a tick attachment rate $>25 \%$ ) were achieved in the control groups of studies 1, 4, 5 and 6 at all tick assessment time points. In study 2 , at the 30 -day assessment time point (4 weeks after treatment), five out of eight control dogs were adequately infested (mean attachment rate $33.0 \%)$ and in study 3 , at the 2 day assessment time point, four out of eight control dogs were adequately infested (mean attachment rate $28.5 \%$ ). However, as these inadequate infestations only occurred on one occasion in each of the two studies and as the mean attachment rate was $>25 \%$, the control dog tick infestations were considered to be sufficiently vigorous to provide a valid result.

All dogs were carefully monitored for adverse events throughout the 12-week post-treatment observation period. One dog in study 2 developed slight erythema at the administration site for $24 \mathrm{~h}$ after treatment, and another dog in study 2 had mild wheal development at the administration site $2 \mathrm{~h}$ after treatment. Both dogs recovered fully without concomitant treatment. No other adverse reactions were observed in these dogs or any other dog in the studies either at the administration site or systemically throughout the study duration.

\section{Discussion}

Fluralaner spot-on solution is the first topically administered ectoparasiticide to provide an extended period of persistent efficacy against ticks and fleas for dogs [14]. Results of the six studies in this paper demonstrate that fluralaner provides excellent therapeutic and persistent efficacy against adult $R h$. sanguineus ticks over the entire 12-week recommended re-treatment interval following single topical administration. These results are consistent with the pharmacokinetic data reported by Kilp et al. [13].

Rh. sanguineus ticks are prevalent in tropical and subtropical regions throughout the entire year and in temperate regions for much of the year $[6,7]$. In addition, these ticks can infest homes and kennels with multiple life stages of ticks present in the housings. Therefore, persistent effective control of this tick is of critical 
Table 2 Mean tick counts and efficacy (\%) after a single topical fluralaner administration (Bravecto ${ }^{\text {TM }}$ spot-on solution) against Rh. sanguineus infestations in dogs

\begin{tabular}{|c|c|c|c|c|c|c|c|c|c|}
\hline \multirow{2}{*}{\multicolumn{2}{|c|}{$\begin{array}{l}\text { Assessment time points }{ }^{a} \\
\text { Study }\end{array}$}} & \multicolumn{2}{|l|}{ Study day 2} & \multicolumn{2}{|c|}{ Study day 30} & \multicolumn{2}{|c|}{ Study day 58} & \multicolumn{2}{|c|}{ Study day 86} \\
\hline & & \multirow{2}{*}{$\begin{array}{c}\text { Fluralaner } \\
1.8(3.8)\end{array}$} & \multirow{2}{*}{$\frac{\text { Control }}{20.5(25.1)}$} & \multirow{2}{*}{$\begin{array}{c}\text { Fluralaner } \\
0(0)\end{array}$} & \multirow{2}{*}{$\frac{\text { Control }}{29.1(29.8)}$} & \multirow{2}{*}{$\begin{array}{c}\text { Fluralaner } \\
0(0)\end{array}$} & \multirow{2}{*}{$\frac{\text { Control }}{32.6(32.9)}$} & \multirow{2}{*}{$\begin{array}{c}\text { Fluralaner } \\
0(0)\end{array}$} & \multirow{2}{*}{$\frac{\text { Control }}{33.8(34.0)}$} \\
\hline 1 & Mean ${ }^{\mathrm{b}}$ tick counts $(n)$ & & & & & & & & \\
\hline & Count range $(n)$ & 0-19 & $4^{e}-44$ & 0 & 20-39 & 0 & $26-39$ & 0 & 29-39 \\
\hline & Efficacy ${ }^{c}(\%)$ & $91.1(84.9)^{d}$ & & $100(100)^{d}$ & & $100(100)^{d}$ & & $100(100)^{d}$ & \\
\hline & $P$-value & 0.0004 & & $<0.0001$ & & $<0.0001$ & & $<0.0001$ & \\
\hline \multirow[t]{4}{*}{2} & Mean ${ }^{\mathrm{b}}$ tick counts $(n)$ & $0(0)$ & $18.7(19.9)$ & $0(0)$ & $13.8(16.5)$ & $0(0)$ & $24.7(26.3)$ & $0.1(0.1)$ & $34.2(35.8)$ \\
\hline & Count range $(n)$ & 0 & $10^{f}-34$ & 0 & $3^{\mathrm{f}}-34$ & 0 & $16-37$ & $0-1$ & $26-67$ \\
\hline & Efficacy $^{\mathrm{C}}(\%)$ & $100(100)^{d}$ & & $100(100)^{d}$ & & $100(100)^{d}$ & & $100(100)^{d}$ & \\
\hline & $P$-value & $<0.0001$ & & $<0.0001$ & & $<0.0001$ & & $<0.0001$ & \\
\hline \multirow[t]{4}{*}{3} & Mean ${ }^{\mathrm{b}}$ tick counts $(n)$ & $0(0)$ & $10.4(14.3)$ & $0(0)$ & $24.3(25.5)$ & $0(0)$ & $15.7(17.9)$ & $0(0)$ & $19.8(23.3)$ \\
\hline & Count range $(n)$ & 0 & $4^{9}-37$ & 0 & $14-38$ & 0 & $6^{9}-26$ & 0 & $6^{9}-35$ \\
\hline & Efficacy ${ }^{c}(\%)$ & $100(100)^{d}$ & & $100(100)^{d}$ & & $100(100)^{d}$ & & $100(100)^{d}$ & \\
\hline & $P$-value & $<0.0001$ & & $<0.0001$ & & $<0.0001$ & & $<0.0001$ & \\
\hline \multirow[t]{4}{*}{4} & Mean ${ }^{\mathrm{b}}$ tick counts $(n)$ & $0.9(2.8)$ & 37.4 (38.6) & $0(0)$ & $26.1(27.7)$ & $0.2(0.3)$ & $18.1(19.6)$ & $0.6(1.1)$ & $19.4(20.8)$ \\
\hline & Count range $(n)$ & $0-23$ & $24-51$ & 0 & $16-41$ & $0-2$ & $8^{h}-33$ & $0-5$ & $12^{\mathrm{h}}-32$ \\
\hline & Efficacy ${ }^{c}(\%)$ & $97.6(92.7)^{d}$ & & $100(100)^{d}$ & & $98.9(98.5)^{d}$ & & $96.7(94.7)^{d}$ & \\
\hline & $P$-value & $<0.001$ & & $<0.001$ & & $<0.001$ & & $<0.001$ & \\
\hline \multirow[t]{4}{*}{5} & Mean ${ }^{\mathrm{b}}$ tick counts $(n)$ & $0.7(1.8)$ & $21.1(25.0)$ & $0(0)$ & $26.4(26.9)$ & $0(0)$ & $28.8(29.8)$ & $0.1(0.1)$ & $22.3(23.1)$ \\
\hline & Count range $(n)$ & $0-11$ & $2^{i}-40$ & 0 & $19-37$ & 0 & $18-43$ & $1-0$ & $13-33$ \\
\hline & Efficacy $^{\mathrm{C}}(\%)$ & $96.6(92.8)^{d}$ & & $100(100)^{d}$ & & $100(100)^{d}$ & & $99.7(99.6)^{d}$ & \\
\hline & $P$-value & $\leq 0.001$ & & $\leq 0.001$ & & $\leq 0.001$ & & $\leq 0.001$ & \\
\hline \multirow[t]{4}{*}{6} & Mean ${ }^{\mathrm{b}}$ tick counts $(n)$ & $0.1(0.1)$ & $30.4(30.8)$ & $0.1(0.1)$ & $24.0(24.5)$ & $0.1(0.1)$ & $17.6(18.7)$ & $0.0(0.0)$ & $17.8(18.3)$ \\
\hline & Count range $(n)$ & $0-1$ & $21-38$ & $0-1$ & $18-31$ & $0-1$ & $12^{j}-33$ & 0 & $11^{\mathrm{j}}-25$ \\
\hline & Efficacy ${ }^{c}(\%)$ & $99.8(99.7)^{d}$ & & $99.7(99.6)^{d}$ & & $99.6(99.5)^{d}$ & & $100(100)^{d}$ & \\
\hline & $P$-value & $<0.001$ & & $<0.001$ & & $<0.001$ & & $<0.001$ & \\
\hline
\end{tabular}

${ }^{a}$ Assessment of ticks $48 \mathrm{~h}\left( \pm 4 \mathrm{~h}\right.$ ) after treatment or re-infestation following treatment; ${ }^{b}$ Geometric mean (arithmetic mean); ${ }^{c}$ Calculated based on geometric mean (arithmetic mean); ${ }^{\mathrm{d}}$ Log-counts of live ticks from the treatment group were significantly different $(P \leq 0.05)$ from log-counts of the respective untreated control group

${ }^{e}$ eight from ten dogs adequately infested; ${ }^{\mathrm{f}}$ seven from eight dogs adequately infested (Study day (SD) 2), five from eight dogs adequately infested (SD 30); ${ }^{9}$ four from eight dogs adequately infested (SD 2), six from eight dogs adequately infested (SD 58), six from eight dogs adequately infested (SD 86); ${ }^{\text {h }}$ eight from ten dogs adequately infested (Study day (SD) 58 and SD 86): ${ }^{i}$ nine from ten dogs adequately infested (Study day (SD) 2); ${ }^{j}$ eight from ten dogs adequately infested (SD58), nine from ten dogs adequately infested (SD86) 
importance. Topically administered fluralaner provides 12 weeks of persistent efficacy against $R h$. sanguineus ticks which means that four treatments will deliver tick protection for approximately 1 year. In comparison, other drugs of the chemical isoxazoline class only provide a maximum of 4-week tick control [11, 12]. The most common cause of treatment failure is reported to be incorrect treatment administration [15], therefore minimizing the number of required administrations can be a factor in reducing the risk of treatment failure.

Topically administered tick repellent treatments may not provide the same level of efficacy in the extremities as can be delivered by systemic treatments [16]. Therefore, topically administration of a systemically active ingredient, in this case fluralaner, provides a high level of efficacy over the entire body surface. As previously noted, $R h$. sanguineus ticks can show attachment predilection for body sites that may not be as effectively protected following topical administration of a non-systemic active product. For example, ticks may attach between the foot pads of dogs on the ventral surface of the foot, an area that is frequently wet and difficult to protect with an external (non-systemic treatment [17]. Bravecto ${ }^{\text {Tx }}$ Spot-on Solution combines convenient administration with persistent efficacy over 12 weeks post-treatment.

Treatment safety is a concern for veterinarians in clinical practice. In the six studies there were mild treatment site dermal reactions observed in two treated dogs that resolved within two or $24 \mathrm{~h}$ without concomitant treatment. There were no systemic adverse reactions in any of the treated dogs. Therefore, it is expected that topically administered fluralaner will deliver the same strong level of systemic safety observed with orally administered fluralaner [18].

\section{Conclusions}

Fluralaner spot-on solution administered once topically to dogs delivers a very high level of therapeutic and persistent efficacy against $R h$. sanguineus ticks over the subsequent 12-weeks.

\section{Competing interests}

MM, JT, AH, and RR are employees of MSD Animal Health. The study was conducted as part of a research program to evaluate the efficacy against $R h$. sanguineus ticks after fluralaner spot-on solution administration. JL, RE, DY, and TV provided contract research support.

\section{Authors' contributions}

The study protocol and report of study 1 was prepared by $J \mathrm{~L}$, for study 2 and 3 by MM, study 4 by WE, study 5 by DY, and study 6 by TV. JT, AH, and RR were responsible for the overall review of the study design, study protocols and reports. FS and EZ provided statistical support for these studies. All authors revised and approved the final version of the manuscript.

\section{Acknowledgements}

The authors would like to express their sincere appreciation for the assistance they received from Melissa A Petersen (Merck US) for studies 4, 5 and 6 . The authors also would like to express their sincere appreciation to the respective teams at each study site, responsible for the animal phase and data collection.

\section{Author details}

MSD Animal Health Innovation GmbH, Zur Propstei, 55270 Schwabenheim, Germany. ${ }^{2}$ ClinVet International, Uitsigweg, Bainsvlei, 9338 Bloemfontein, Free State, South Africa. ${ }^{3}$ MSD Animal Health Malelane Research Unit, PO Box 124, Malalane 1320, Republic of South Africa. ${ }^{4}$ BerTek, Inc., 104 Wilson Bottoms Road, Greenbrier, AR 72058, USA. ${ }^{5}$ Young Veterinary Research Services, 7243 East Avenue, Turlock, CA 95380, USA. ${ }^{6}$ MPI Research, 54943 North Main Street, Mattawan, MI 49071, USA. ${ }^{7}$ Merck Animal Health, 2 Giralda Farms, Madison, NJ 07940-1026, USA.

Received: 24 February 2016 Accepted: 20 April 2016 Published online: 31 May 2016

\section{References}

1. Dantas-Torres F. Biology and ecology of the brown dog tick, Rhipicephalus sanguineus. Parasit Vectors. 2007;3:26.

2. Szabo MP, Cunha TM, Pinter A, Vicentini F. Ticks (Acari: Ixodidae) associated with domestic dogs in Franca region, Sao Paulo, Brazil. Exp Appl Acarol. 2001;25:909-16.

3. Dantas-Torres F, Melo MF, Figueredo LA, Brandao-Filho SP. Ectoparasite infestation on rural dogs in the municipality of Sao Vicente Ferrer, Pernambuco, Northeastern Brazil. Rev Bras Parasitol Vet. 2009:18:75-7.

4. Dantas-Torres F, Figueredo LA, Brandao-Filho SP. Rhipicephalus sanguineus (Acari: Ixodidae), the brown dog tick, parasitizing humans in Brazil. Rev Soc Bras Med Trop. 2006;39:64-7.

5. Dantas-Torres F, Ferreira DR, de Melo LM, Lima PA, Siqueira DB, Rameh-deAlbuquerque LC, de Melo AV, Ramos JA. Ticks on captive and free-living wild animals in northeastern Brazil. Exp Appl Acarol. 2009;50:181-9.

6. Koch HG. Seasonal incidence and attachment sites of ticks (Acari: Ixodidae) on domestic dogs in southeastern Oklahoma and northwestern Arkansas, USA. J Med Entomol. 1982;19:293-8.

7. Jacobs PA, Fourie LJ, Kok DJ, Horak IG. Diversity, seasonality and sites of attachment of adult ixodid ticks on dogs in the central region of the Free State Province, South Africa. Onderstepoort J Vet Res. 2001;68:281-90.

8. Taylor MA. Recent developments in ectoparasiticides. Vet J. 2001;161:253-68.

9. Varloud M, Fourie JJ. One-month comparative efficacy of three topical ectoparasiticides against adult brown dog ticks (Rhipicephalus sanquineus sensu lato) on mixed-bred dogs in controlled environment. Parasitol Res. 2015;114:1711-9.

10. Gassel M, Wolf C, Noack S, Williams H, Ilg T. The novel isoxazoline ectoparasiticide fluralaner: selective inhibition of arthropod gammaaminobutyric acid- and L-glutamate-gated chloride channels and insecticidal/acaricidal activity. Insect Biochem Mol Biol. 2012;45:111-24.

11. Shoop WL, Hartline EJ, Gould BR, Waddell ME, McDowell RG, Kinney JB, Lahm GP, Long JK, Xu M, Wagerle T, et al. Discovery and mode of action of afoxolaner, a new isoxazoline parasiticide for dogs. Vet Parasitol. 2014;201:179-89.

12. McTier T, Chubb N, Curtis M, Hedges L, Inskeep G, Knauer C, Menon S, Mills B, Pullins A, Zinser E, et al. Discovery of sarolaner: A novel, orally administered, broad-spectrum, isoxazoline ectoparasiticide for dogs. Vet Parasitol 2016: http://dx.doi.org/10.1016/j.vetpar.2016.02.019. Accessed 30 May 2016.

13. Kilp S, Ramirez D, Allan M, Roepke R. Comparative pharmacokinetics of fluralaner in dogs and cats following single topical or intravenous administration. Parasit Vectors 2016, in press.

14. European Commission: Community register of veterinary medicinal products, Product information: Bravecto Spot-on Solution: http://www.ema.europa.eu/ ema/index.ssp?curl=pages/medicines/veterinary/medicines/002526/vet_med_ 000285.jsp\&mid=WC0b01ac058001fa1c. Accessed 30 May 2016.

15. Coles TB, Dryden MW. Insecticide/acaricide resistance in fleas and ticks infesting dogs and cats. Parasit Vectors. 2014;7:8.

16. Pfister K, Armstrong R. Efficacy of systemically and/or topically distributed ectoparasiticides in dogs: a literature-based review related to fluralaner and permethrin as examples. Parasite Vectors 2016, in press.

17. Lussenhop J, Baumer W, Kietzmann M, Schnieder T, Wolken S. Dynamics of distribution and efficacy of different spot-on permethrin formulations in dogs artificially infested with Dermacentor reticulatus. Parasit Vectors. 2011;4:45.

18. European Commission: Community register of veterinary medicinal products, Product information: Bravecto: Annex 1 Summary of Product Characteristics: http://www.ema.europa.eu/ema/index.jsp?curl=pages/ medicines/veterinary/medicines/002526/vet_med_000285.jsp\&mid= WC0b01ac058001fa1c. Accessed 30 Apr 2015. 